\title{
CIRCUMSTELLAR STRUCTURE AROUND EVOLVED STARS IN THE CYGNUS-X STAR FORMATION REGION
}

\author{
Kathleen E. Kraemer ${ }^{1}$, Joseph L. Hora ${ }^{2}$, Michael P. Egan ${ }^{3}$, Joseph Adams ${ }^{4}$, Lori E. Allen ${ }^{5}$, Sylvain Bontemps ${ }^{6}$, \\ Sean J. Carey ${ }^{7}$, Giovanni G. Fazio ${ }^{2}$, Robert Gutermuth ${ }^{8,9}$, Eric Keto ${ }^{2}$, Xavier P. Koenig $^{2}$, S. Thomas Megeath $^{10}$, \\ Donald R. Mizuno ${ }^{11}$, Frederique Motte ${ }^{12}$, Stephan D. Price ${ }^{1}$, Nicola Schneider ${ }^{12}$, Robert Simon ${ }^{13}$, and \\ HowARD A. SMITH ${ }^{2}$ \\ ${ }^{1}$ Air Force Research Laboratory, Space Vehicles Directorate, 29 Randolph Road, Hanscom AFB, MA 01731, USA; afrl.rvb.pa@hanscom.af.mil \\ ${ }^{2}$ Harvard-Smithsonian Center for Astrophysics, 60 Garden Street, MS 65, Cambridge, MA 02138, USA; jhora@ cfa.harvard.edu, gfazio@cfa.harvard.edu, \\ eketo@cfa.harvard.edu, xkoenig@cfa.harvard.edu, hsmith@cfa.harvard.edu \\ ${ }^{3}$ National Geo-Spatial Intelligence Agency, DN-11, 12310 Sunrise Valley Drive, Reston, VA 20191, USA; michael.p.egan@nga.mil \\ ${ }^{4}$ Cornell University, NY, USA; jdadams@astro.cornell.edu \\ ${ }^{5}$ National Optical Astronomy Observatory, Tucson, AZ, USA; lallen@ noao.edu \\ ${ }^{6}$ Observatoire de Bordeaux, Floirac, France; Sylvain.Bontemps@obs.u-bordeaux1.fr \\ ${ }^{7}$ Spitzer Science Center, Caltech, MS 220-6, Pasadena, CA 91125, USA; carey@ipac.caltech.edu \\ ${ }^{8}$ Five College Astronomy Department, Smith College, Northampton, MA 01063, USA; rgutermu@ smith.edu \\ ${ }^{9}$ Department of Astronomy, University of Massachusetts, Amherst, MA 01003, USA \\ ${ }^{10}$ Department of Physics and Astronomy, University of Toledo, Toledo, OH 43606, USA; meageath@ astro1.panet.utoledo.edu \\ ${ }^{11}$ Institute for Scientific Research, Boston College, Chestnut Hill, MA 02467-3800, USA; afrl.rvb.pa@ hanscom.af.mil \\ ${ }^{12}$ Commissariat à l'energie atomique, Saclay, Paris, France; motte@ discovery.saclay.cea.fr, nschneid@cea.fr \\ ${ }^{13}$ Universität zu Köln, Köln, Germany; simonr@ph1.uni-koeln.de \\ Received 2009 October 7; accepted 2010 March 8; published 2010 April 23
}

\begin{abstract}
We present observations of newly discovered $24 \mu \mathrm{m}$ circumstellar structures detected with MIPS around three evolved stars in the Cygnus-X star-forming region. One of the objects, BD+43 3710, has a bipolar nebula, possibly due to an outflow or a torus of material. A second, HBHA 4202-22, a Wolf-Rayet candidate, shows a circular shell of $24 \mu \mathrm{m}$ emission suggestive of either a limb-brightened shell or disk seen face-on. No diffuse emission was detected around either of these two objects in the Spitzer 3.6-8 $\mu \mathrm{m}$ IRAC bands. The third object is the luminous blue variable candidate G79.29+0.46. We resolved the previously known inner ring in all four IRAC bands. The $24 \mu \mathrm{m}$ emission from the inner ring extends $\sim 1.2$ beyond the shorter wavelength emission, well beyond what can be attributed to the difference in resolutions between MIPS and IRAC. Additionally, we have discovered an outer ring of $24 \mu \mathrm{m}$ emission, possibly due to an earlier episode of mass loss. For the two shell stars, we present the results of radiative transfer models, constraining the stellar and dust shell parameters. The shells are composed of amorphous carbon grains, plus polycyclic aromatic hydrocarbons in the case of G79.29+0.46. Both G79.29+0.46 and HBHA 4202-22 lie behind the main Cygnus-X cloud. Although G79.29+0.46 simply may be on the far side of the cloud, HBHA 4202-22 is unrelated to the Cygnus-X star formation region.
\end{abstract}

Key words: stars: AGB and post-AGB - stars: individual (BD+43 3710, HBHA 4202-22, G79.29-0.46)

\section{INTRODUCTION}

Cygnus- $\mathrm{X}$ is arguably the biggest and brightest massive star formation region within $2 \mathrm{kpc}$ of the Sun $(d \sim 1.7 \mathrm{kpc}$; Schneider et al. 2006, 2007). It contains several hundred H II regions, numerous $\mathrm{OB}$ associations with over a thousand $\mathrm{OB}$ stars, and thousands of low-mass stars. We have performed an unbiased survey of $\sim 24 \mathrm{deg}^{2}$ in Cygnus-X with IRAC (Fazio et al. 2004) and MIPS (Rieke et al. 2004) on the Spitzer Space Telescope (Werner et al. 2004). The primary objectives of this Spitzer Legacy project were to study the evolution of highmass protostars, clustering of both high- and low-mass stars in the complex, as well as several other goals described by Hora et al. (2008b). Follow-up spectroscopy with Spitzer's Infrared Spectrograph (IRS; Houck et al. 2004) was obtained for several dozen promising sources in the MIPS and IRAC survey regions.

In addition to the numerous young stars that are the primary focus of the Legacy project, there are dozens of evolved objects in the survey region, from Miras and Cepheids to Wolf-Rayet (WR) and carbon stars to planetary nebulae (PNe) and supernova remnants (SNRs). After the initial discovery of a bipolar nebula at $24 \mu \mathrm{m}$ around a carbon star during the data processing quality control effort, a systematic search for additional circumstellar nebulae was made. SIMBAD was used to compile positional lists of evolved objects that lie within the bounds of the $24 \mu \mathrm{m}$ observations. The $24 \mu \mathrm{m}$ images were then inspected for the presence of point sources and associated nebulosity, if any. More than $80 \%$ (146) of the 191 known evolved objects are detected at $24 \mu \mathrm{m}$ as point sources (Table 1). These types of objects can be bright in the infrared for a variety of reasons such as emission from dust or forbidden lines. This, of course, is an incomplete survey of the evolved stars as it does not include the faint population of asymptotic giant branch (AGB) stars detected with Spitzer (also known as contaminants in the young stellar object studies). Be that as it may, only three evolved stars in the Cygnus- $X$ region were distinguished from the vast majority by exhibiting extended $24 \mu \mathrm{m}$ emission. The emission around two of these stars, which are listed in Table 2, was first reported by Kraemer et al. (2009). Here, we discuss the morphological characteristics of the circumstellar structures as well as the spectral properties of the dust and the central exciting objects. 
Table 1

Evolved Objects in Cygnus-X: Point-source Detections at $24 \mu \mathrm{m}$

\begin{tabular}{lccc}
\hline \hline \multicolumn{1}{c}{$\begin{array}{c}\text { Object } \\
\text { Type }\end{array}$} & $\begin{array}{c}\text { No. in } 24 \mu \mathrm{m} \\
\text { Region }\end{array}$ & $\begin{array}{c}\text { Detected at } 24 \mu \mathrm{m} \\
\text { Region }\end{array}$ & $\begin{array}{c}\text { Detection } \\
\text { Rate }\end{array}$ \\
\hline PNe & 7 & 5 & $71 \%$ \\
WRs & 9 & 9 & $100 \%$ \\
Post-AGBs & 2 & 2 & $100 \%$ \\
Carbon stars & 52 & 51 & $98 \%$ \\
S stars & 6 & 5 & $83 \%$ \\
SNRs & $5 ?$ & $0 ?$ & $0 ? \%$ \\
Miras & 4 & 4 & $100 \%$ \\
Semi-regulars & 4 & 4 & $100 \%$ \\
Cepheids & 4 & 4 & $100 \%$ \\
Dwarf novae & 3 & 1 & $33 \%$ \\
Other V*s & 85 & 61 & $72 \%$ \\
\hline
\end{tabular}

Notes. Object type as reported in Simbad. This is likely to be an incomplete listing, since not all objects are typed correctly. BD+43 3710, for instance, is listed merely as a star. There were an additional 40 variable stars, mostly generic "V*" type but including one RR Lyr, which had insufficiently precise coordinates to determine if they had $24 \mu \mathrm{m}$ counterparts. It is not clear how big the SNRs are expected to be at $24 \mu \mathrm{m}$, so the data inspection may not have been sensitive to their emission.

\section{OBSERVATIONS AND DATA REDUCTION}

The Cygnus-X Legacy Survey (Hora et al. 2008b) observed a $\sim 7^{\circ} \times 7^{\circ}$ region centered on $(l, b) \sim\left(80^{\circ}, 0 \circ 3\right)$ with Spitzer's IRAC (all four bands) and MIPS (24 and $70 \mu \mathrm{m}$ ). The MIPS observations followed the MIPSGAL survey strategy (Carey et al. 2009), using the fast-scanning mode with $3 \mathrm{~s}$ integrations and $148^{\prime \prime}$ between legs. This results in $\sim 10$ times redundancy per pixel or $\sim 30 \mathrm{~s}$ total integration time. We use the $24 \mu \mathrm{m}$ data processing pipeline optimized for MIPSGAL, which is based on the Spitzer Science Center (SSC) pipeline ( S16.1). It includes the standard "read-2," row-droop, and droop corrections, dark subtraction, flat fielding, and a modified linearization. The optimization involves better artifact mitigation such as overlap, jailbar, and latent corrections; additional details are described by Mizuno et al. (2008) and Carey et al. (2009). The $70 \mu \mathrm{m}$ data are not considered here and thus are not described. For completeness, we note that BD+43 3710 was outside the $70 \mu \mathrm{m}$ coverage, HBHA 4202-22 is not detected in the post-bcd images, and the inner ring of G79.29+0.46 is apparent in the post-bcd images but the new outer ring is not (see Section 3.3).

The Cygnus- $X$ survey measurements with IRAC consisted of $\sim 1$. $1 \times 1$. 1 tiles taken in high dynamic range mode with $3 \times 12 \mathrm{~s}$ integrations. The IRAC data were processed using the SSC pipeline S16.1 products with artifact mitigation performed for jailbar, pulldown, muxbleed, and banding effects from bright sources. Mosaics were created with the WCSmosaic package which performs additional corrections detailed by Gutermuth et al. (2008). Photometry was extracted using the "PhotVis" package (Gutermuth et al. 2008) for the IRAC data and the
MIPSGAL point-source pipeline (S. Shenoy et al. 2010, in preparation) for the $24 \mu \mathrm{m}$ data.

BD+43 3710, discovered as being extended in the MIPS data taken in 2007 December, was outside the IRAC coverage and so was observed in a separately designed Astronomical Observation Request (AOR). The same data reduction process was applied as with the primary IRAC observations. HBHA 4202-22 was at the edge of the IRAC coverage region and was observed only with the 3.6 and $5.8 \mu \mathrm{m}$ arrays, as the circumstellar ring was not discovered in time to adjust the AORs or add an additional field as was done for BD+43 3710 .

We also observed BD+43 3710 with the IRS, targeting the central star and one position in each lobe of the $24 \mu \mathrm{m}$ bipolar nebula. The observations used all four low-resolution modes and the data (Pipeline S18.0.2) were reduced using the Spitzer IRS Custom Extraction (SPICE) software, version 2.1.2, provided by the SSC. For the central star observations, we subtracted the alternate order for the short-low (SL) data and the alternate nod for the long-low (LL) data prior to performing the pointsource extraction. After the spectra were extracted with SPICE, they were trimmed to get rid of bad data at the extremes of the wavelength ranges and averaged together. For the positions in the lobes, we used the full slit extraction, which includes corrections to the calibration due to the aperture size and extended nature of the target. There were no compact sources apparent in either the SL or LL slit for the lobe observations; no background subtraction was performed. The luminous blue variable (LBV) candidate G79.29+0.46 was observed with IRS in high-resolution mode by another group and results are presented by G. Umana et al. (2010, in preparation).

Photometric measurements from this work and from the literature are summarized in Table 3 for each of the three stars. BVR data are from the Naval Observatory Merged Astrometric Dataset (NOMAD; Zacharias et al. 2004), JHK data are from the Two Micron All-Sky Survey (2MASS; Cutri et al. 2003), and mid-infrared data are from our Spitzer measurements. The uncertainties for the Spitzer data are the extraction-based uncertainties combined with the absolute uncertainties for IRAC (2\%; Hora et al. 2008a) and MIPS (4\%; Engelbracht et al. 2007). Those for 2MASS are from Cutri et al. (2003; see also Skrutskie et al. 2006); the BVR uncertainties are estimated at $4 \%$ as they are not listed in NOMAD.

\section{RESULTS AND DISCUSSION}

\section{1. $B D+433710$}

BD+43 3710, located in the extreme northeast corner of the Cygnus- $\mathrm{X}$ survey region, is a little known carbon star candidate classified as spectral type R: by Nassau \& Blanco (1954). It shows a remarkable bipolar structure at $24 \mu \mathrm{m}$ that is completely absent at the shorter wavelengths (it is also outside the $70 \mu \mathrm{m}$ MIPS coverage). Figure 1 shows a three-color image of BD+43

Table 2

Source Parameters

\begin{tabular}{|c|c|c|c|c|c|c|}
\hline Name & R.A. (J2000) & Decl. (J2000) & $l$ & $b$ & $\begin{array}{c}\text { Spectral } \\
\text { Type }\end{array}$ & $\begin{array}{c}\text { Object } \\
\text { Type }\end{array}$ \\
\hline $\mathrm{BD}+433710$ & $20^{\mathrm{h}} 45^{\mathrm{m}} 34^{\mathrm{s}} .73$ & $+43^{\circ} 32^{\prime} 27^{\prime \prime} .28$ & 83.3755 & 0.3400 & $\mathrm{R}:$ & Star \\
\hline НBHA 4202-22 & $20^{\mathrm{h}} 17^{\mathrm{m}} 8^{\mathrm{s}} .12$ & $+41^{\circ} 07^{\prime} 27^{\prime \prime} .0$ & 78.3203 & 3.1536 & WR:, A0I & Em. line stal \\
\hline G79.29+0.46 & $20^{\mathrm{h}} 31^{\mathrm{m}} 42^{\mathrm{s}} .28$ & $+40^{\circ} 21^{\prime} 59^{\prime \prime} .1$ & 80.9005 & 1.6435 & $\mathrm{~B}[\mathrm{e}] \mathrm{I}:$ & Star \\
\hline
\end{tabular}

Notes. Object types as reported in Simbad. Spectral types are from Nassau \& Blanco (1954), Kohoutek \& Wehmeyer (1999), and this work, and Voors et al. (2000) for BD+43 3710, HBHA 4202-22, and G79.29+0.46, respectively, although see the text for discussion. 
Table 3

Source Fluxes

\begin{tabular}{|c|c|c|c|c|c|c|c|c|c|c|c|}
\hline Source & $B$ & $V$ & $R$ & $J$ & $H$ & $K$ & [3.6] & [4.5] & [5.8] & [8.0] & [24] \\
\hline $\mathrm{BD}+433710$ & $\begin{array}{c}11.22 \pm 0.04 \mathrm{mag} \\
0.135 \mathrm{Jy}\end{array}$ & $\begin{array}{c}10.06 \pm 0.04 \\
0.334\end{array}$ & $\begin{array}{c}9.35 \pm 0.04 \\
0.537\end{array}$ & $\begin{array}{c}6.60 \pm 0.02 \\
3.66\end{array}$ & $\begin{array}{c}6.14 \pm 0.02 \\
3.57\end{array}$ & $\begin{array}{c}5.88 \pm 0.02 \\
2.96\end{array}$ & $\begin{array}{c}5.76 \pm 0.02 \\
1.40\end{array}$ & $\begin{array}{c}5.63 \pm 0.02 \\
1.01\end{array}$ & $\begin{array}{c}5.54 \pm 0.02 \\
0.70\end{array}$ & $\begin{array}{c}5.45 \pm 0.02 \\
0.42\end{array}$ & $\begin{array}{c}5.09 \pm 0.05 \\
0.07\end{array}$ \\
\hline HBHA 4202-22 & $\begin{array}{c}16.54 \pm 0.04 \\
0.001\end{array}$ & $\begin{array}{c}15.18 \pm 0.04 \\
0.003\end{array}$ & $\begin{array}{c}14.53 \pm 0.04 \\
0.005\end{array}$ & $\begin{array}{c}10.15 \pm 0.02 \\
0.139\end{array}$ & $\begin{array}{c}9.27 \pm 0.02 \\
0.202\end{array}$ & $\begin{array}{c}8.65 \pm 0.02 \\
0.231\end{array}$ & $\begin{array}{c}7.89 \pm 0.02 \\
0.196\end{array}$ & $\begin{array}{l}\cdots \\
\cdots\end{array}$ & $\begin{array}{c}7.20 \pm 0.02 \\
0.151\end{array}$ & $\begin{array}{l}\cdots \\
\cdots\end{array}$ & $\begin{array}{c}5.39 \pm 0.05 \\
0.050\end{array}$ \\
\hline G79.29+0.46 & $\begin{array}{c}19.30 \pm 0.04 \\
7.9(-5)\end{array}$ & $\begin{array}{c}16.73 \pm 0.04 \\
7.2(-4)\end{array}$ & $\begin{array}{c}15.20 \pm 0.04 \\
2.5(-3)\end{array}$ & $\begin{array}{c}6.91 \pm 0.03 \\
2.73\end{array}$ & $\begin{array}{c}5.29 \pm 0.02 \\
7.81\end{array}$ & $\begin{array}{c}4.33 \pm 0.02^{\mathrm{a}} \\
12.37\end{array}$ & $\begin{array}{l}\cdots \\
\cdots\end{array}$ & $\begin{array}{l}\cdots \\
\cdots\end{array}$ & $\begin{array}{c}3.07 \pm 0.02^{\mathrm{a}} \\
6.82\end{array}$ & $\begin{array}{c}2.86^{\mathrm{a}} \\
4.61\end{array}$ & $\begin{array}{c}2.05 \pm 0.04^{\mathrm{a}} \\
1.09\end{array}$ \\
\hline
\end{tabular}

Note. ${ }^{\text {a }}$ Possibly saturated, so the uncertainties are undoubtedly underestimated. 


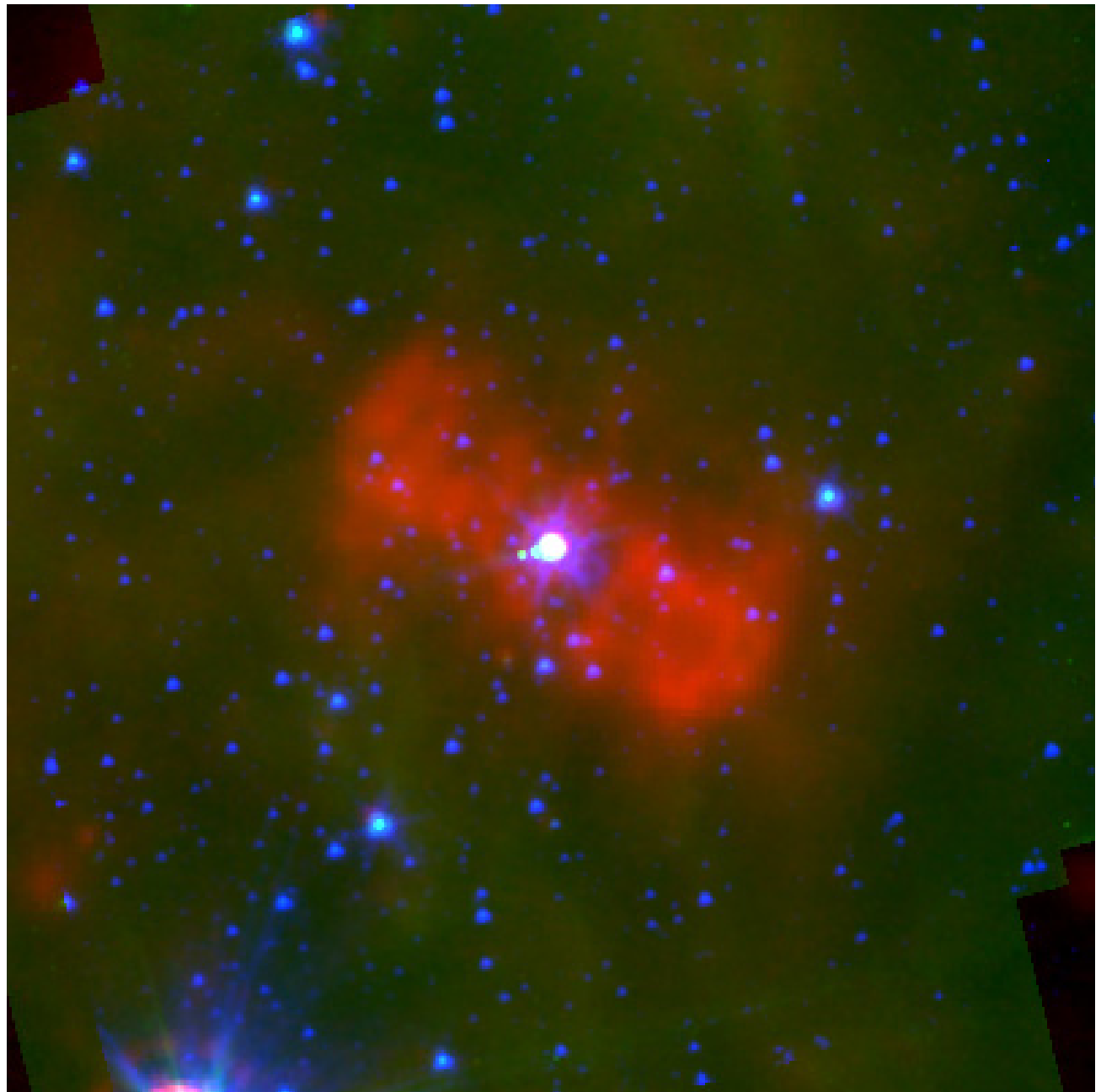

Figure 1. Three-color image of BD+43 3710. Red: $24 \mu \mathrm{m}$; green: $8 \mu \mathrm{m}$; blue: $3.6 \mu \mathrm{m}$. The image is $5.78 \times 5.78$, or $\sim 2.9 \times 2.9 \mathrm{pc}$, assuming a distance of $1.7 \mathrm{kpc}$.

3710 combining the $3.6 \mu \mathrm{m}, 8 \mu \mathrm{m}$, and $24 \mu \mathrm{m}$ data, while Figure 2 shows the individual images from all four IRAC bands plus the MIPS $24 \mu \mathrm{m}$ image. The $24 \mu \mathrm{m}$ emission is roughly $2.7 \times 0.9$ or $\sim 1.34 \times 0.45 \mathrm{pc}$, assuming the Cygnus- $\mathrm{X}$ distance of $1.7 \mathrm{kpc}$.

The central star is in the Bonner Durchmusterung (Argelander 1903), Tycho (Høg et al. 2000), 2MASS (Cutri et al. 2003), and Midcourse Space Experiment (MSX; Egan et al. 2003) point-source catalogs. However, no references discussing its physical properties could be found in the literature. The visual magnitude did change from a reported $9.2 \mathrm{mag}$ in the Bonner Durchmusterung (Argelander 1903) to $10.1 \mathrm{mag}$ in the early 1990s as measured by Tycho. Other than indicating variability in this object, which is not an unusual feature in a carbon star, such a sparse sampling of the temporal baseline (albeit long) does not allow us to say anything meaningful about the pulsation properties of the star. Figure 3 shows the spectral energy distribution (SED) for BD+43 3710, as well as the other two sources. The smooth curves are Planck curve fits to the available photometry. A single-temperature graybody does not well represent the emission from any of these sources, although the BVR data are probably suppressed by an unknown amount of extinction.

As mentioned above, we obtained low-resolution IRS data on BD+43 3710 and one position in each lobe. Figure 4 (left) shows the locations where the spectra were taken. The right side shows the resulting spectrum for the central star. The spectrum is largely featureless. In comparison to the naked stars in the ISOSWS atlas of Sloan et al. (2003), it is most similar to HD 19557, a carbon star of type R noted by Goebel et al. (1983) as having particularly weak carbon features in visible and nearIR bands. In particular, the inflection in the IRS spectrum at $9 \mu \mathrm{m}$ is well matched in the SWS spectrum of HD 19557, although it is not apparent in most of the naked carbon stars in the SWS atlas (class 1.NC of Kraemer et al. 2002) or in the other dust-free SWS spectral classes (1.N, 1.NO, 1.NE, and 1.NM). The IRS spectrum, however, does not show the turnover at $\sim 5.5 \mu \mathrm{m}$ expected from $\mathrm{C}_{3}, \mathrm{CO}$, and $\mathrm{CN}$ absorption features (Goebel et al. 1978) typically observed in carbon stars (e.g., Aoki et al. 1998; Zijlstra et al. 2006). Several hydrogen recombination lines are detected in emission, as annotated in the figure. The strengths of the $\operatorname{Pf} \alpha, \mathrm{Hu} \alpha$, and $\mathrm{H}$ I 8-7 do not change between the raw data and the background-subtracted data, unlike the typical nebular lines such as the [Ne II] feature at $12.8 \mu \mathrm{m}$, which nearly vanishes after background subtraction. Thus, the hydrogen recombination lines are likely associated with BD+43 3710 and its nebula, and are not simply in the foreground or background cloud. Although not typical for carbon stars, Balmer lines have been detected in emission in the carbon star UV Aur A (Herbig 2009). Thus, we find that the carbon star spectral classification for BD+43 3710 is supported by the IRS spectrum but not definitively confirmed. 


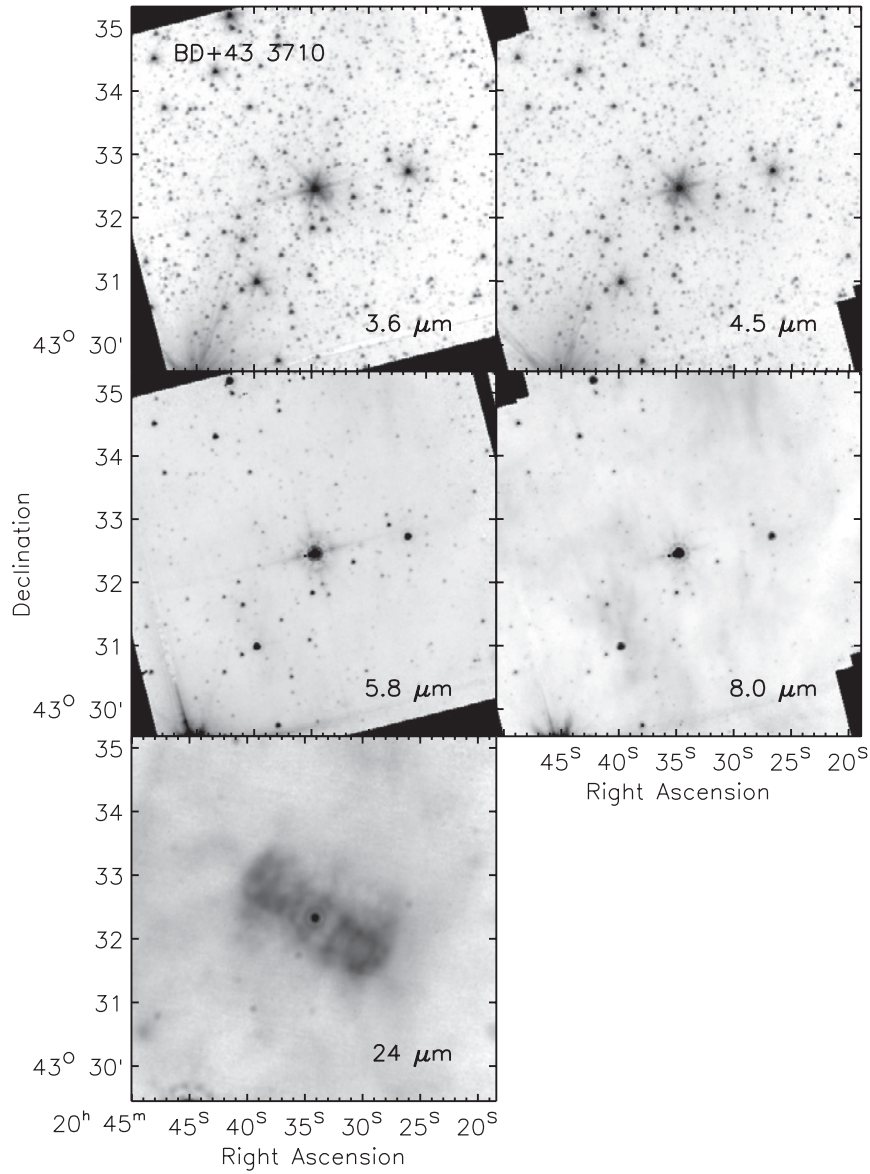

Figure 2. Individual images of BD+43 3710 in the IRAC bands (top two rows) and in MIPS $24 \mu \mathrm{m}$ (bottom image). Grayscales are logarithmic.

The spectra from the two lobes, shown in Figure 5, are quite similar to each other, with a cool dust spectrum that rises with wavelength past the end of the IRS band and a few broad features and low-excitation fine-structure lines. The SED of the western lobe rises a bit more steeply with wavelength than that of the eastern lobe, possibly indicative of slightly warmer dust. Neither spectrum is consistent with a single-temperature graybody, but the majority of the dust must be cooler than $\sim 100 \mathrm{~K}$ since the SED peaks beyond $35 \mu \mathrm{m}$. A $108 \mathrm{~K}$ graybody is the "best" fit to the LL data for both lobes and is shown in the figure for comparison. As can be seen from the spectra, unlike in a small number of sources where the circumstellar emission in the MIPS $24 \mu \mathrm{m}$ band has been found to arise from a high excitation [O IV] line (e.g., Morris et al. 2004 (a WN star); Morris et al. 2006 (an SNR candidate); Billot et al. 2009 (sources of unknown nature, possibly SNR or PNe candidates); N. Flagey et al. 2010, in preparation), or the $\sim 26-30 \mu \mathrm{m} \mathrm{MgS}$ feature seen in some post-AGB stars and PNe (e.g., Forrest et al. 1981; Goebel \& Moseley 1985; Hony et al. 2002; BernardSalas et al. 2009), the nebular emission around BD+43 3710 arises from small dust grains. They are probably carbon-rich grains, dominated by amorphous carbon, given the lack of either a silicate emission feature around $9.7 \mu \mathrm{m}$ or silicon carbide around $11 \mu \mathrm{m}$. The broad features present in the spectra at $6.3,7-8,11.3$, and $16-18 \mu \mathrm{m}$ typically attributed to polycyclic aromatic hydrocarbons (PAHs), are not likely to be from the BD+43 3710 nebula since there is no corresponding nebulosity in the IRAC 5.8 or $8.0 \mu \mathrm{m}$ image that can be readily associated with the $24 \mu \mathrm{m}$ lobes (Figure 2). The PAHs and low-energy
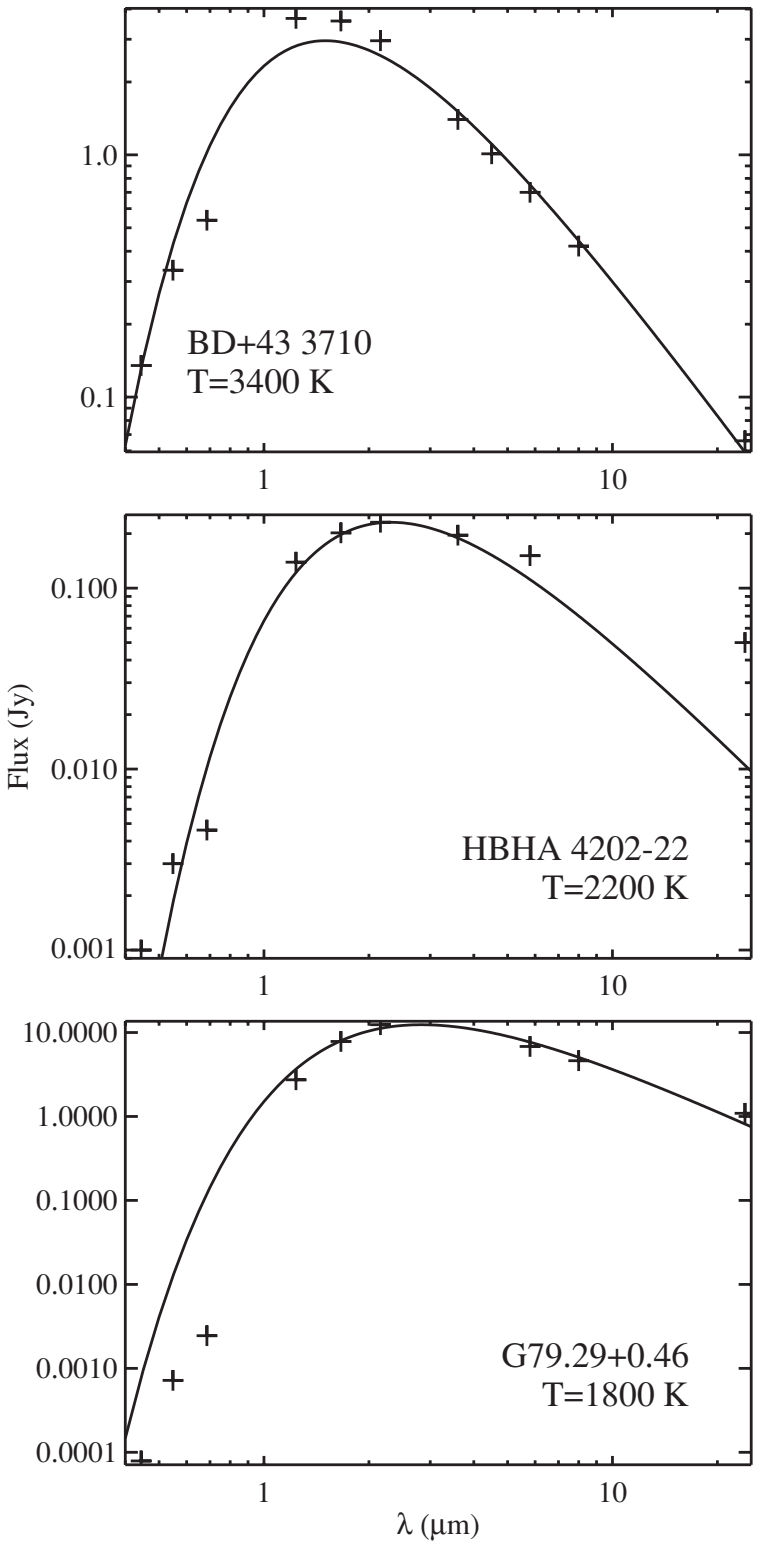

Figure 3. SEDs for BD+43 3710 (top), HBHA 4202-22 (middle), and G79.29+0.46 (bottom). Temperatures for the best-fit (minimal $\chi^{2}$ ) graybody curve (smooth curves) are given in the lower left of each panel. Pluses correspond to the photometry from Table 3. Uncertainties are smaller than the symbol size.

fine-structure lines seen in Figure 5 are extremely common in active star-forming regions such as Cygnus-X, so those features likely arise from the fore/background Cygnus-X cloud.

\subsection{HBHA 4202-22}

HBHA 4202-22 is located on the western edge of the survey region. Kohoutek \& Wehmeyer (1999) report $\mathrm{H} \alpha$ emission from the star, citing "Dolidze (1971) No. 3" which probably corresponds to Dolidze (1971). This may be where the WR candidacy given in SIMBAD comes from although we could not confirm this due to the unavailability of the Russian circular, and it is otherwise unnoted in the literature. Figure 6 shows a threecolor image from the 3.6, 5.8, and $24 \mu \mathrm{m}$ data, and the individual images are in Figure 7. We cannot say definitively there is no emission from PAHs, as we do not have the $8 \mu \mathrm{m}$ data which would contain the (typically) strongest feature. However, there 

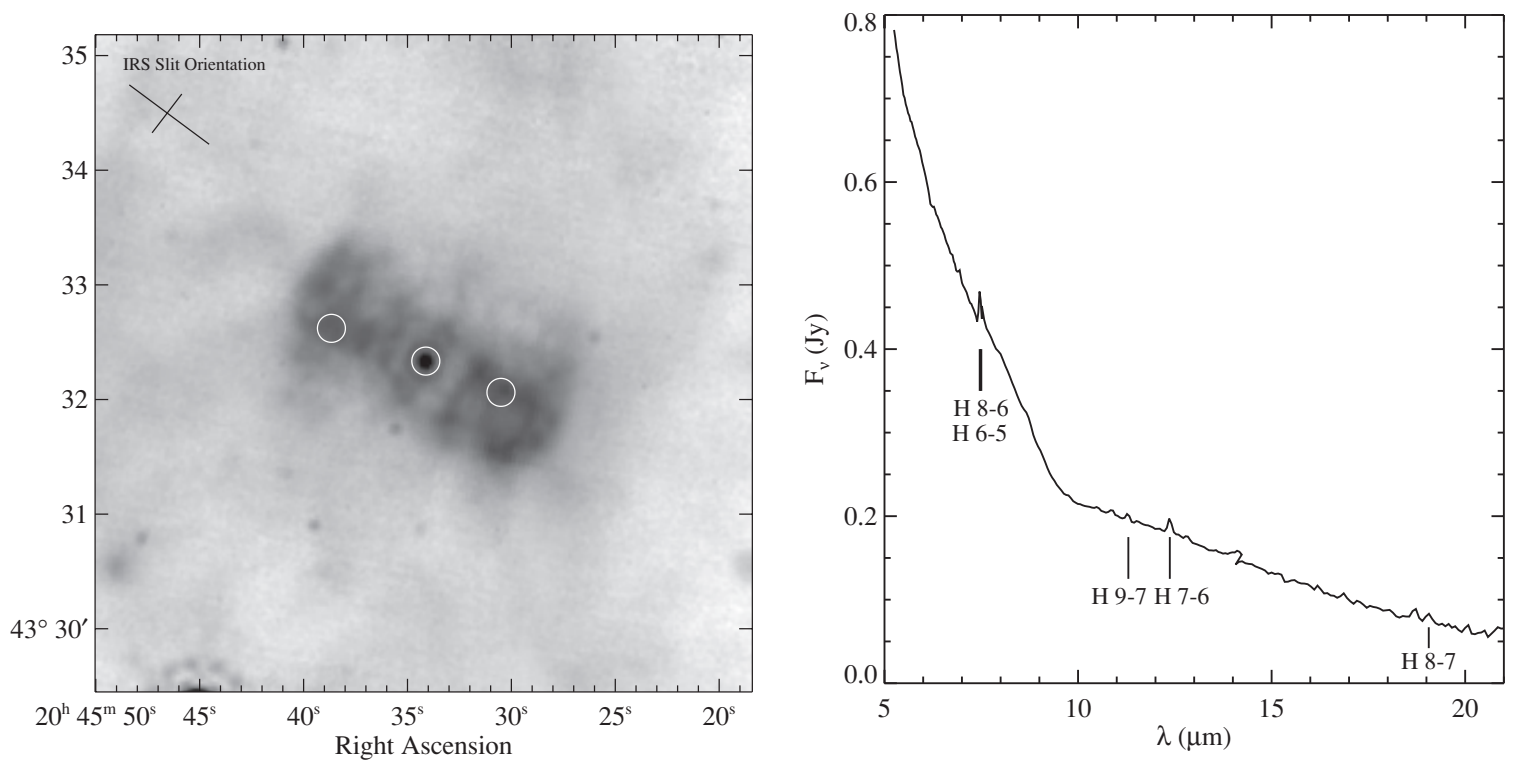

Figure 4. Left: positions (circles) observed with IRS in BD+43 3710, overlaid on the $24 \mu \mathrm{m}$ data (grayscale). The orientation of the SL and LL slits is shown in the upper left corner. Right: IRS 5.25-21 $\mu \mathrm{m}$ spectrum of BD+43 3710. A handful of hydrogen recombination lines are detected in emission and are labeled on the plot. The feature slightly shortward of $\mathrm{H} 8-7$ is only present in one nod and so is probably spurious.
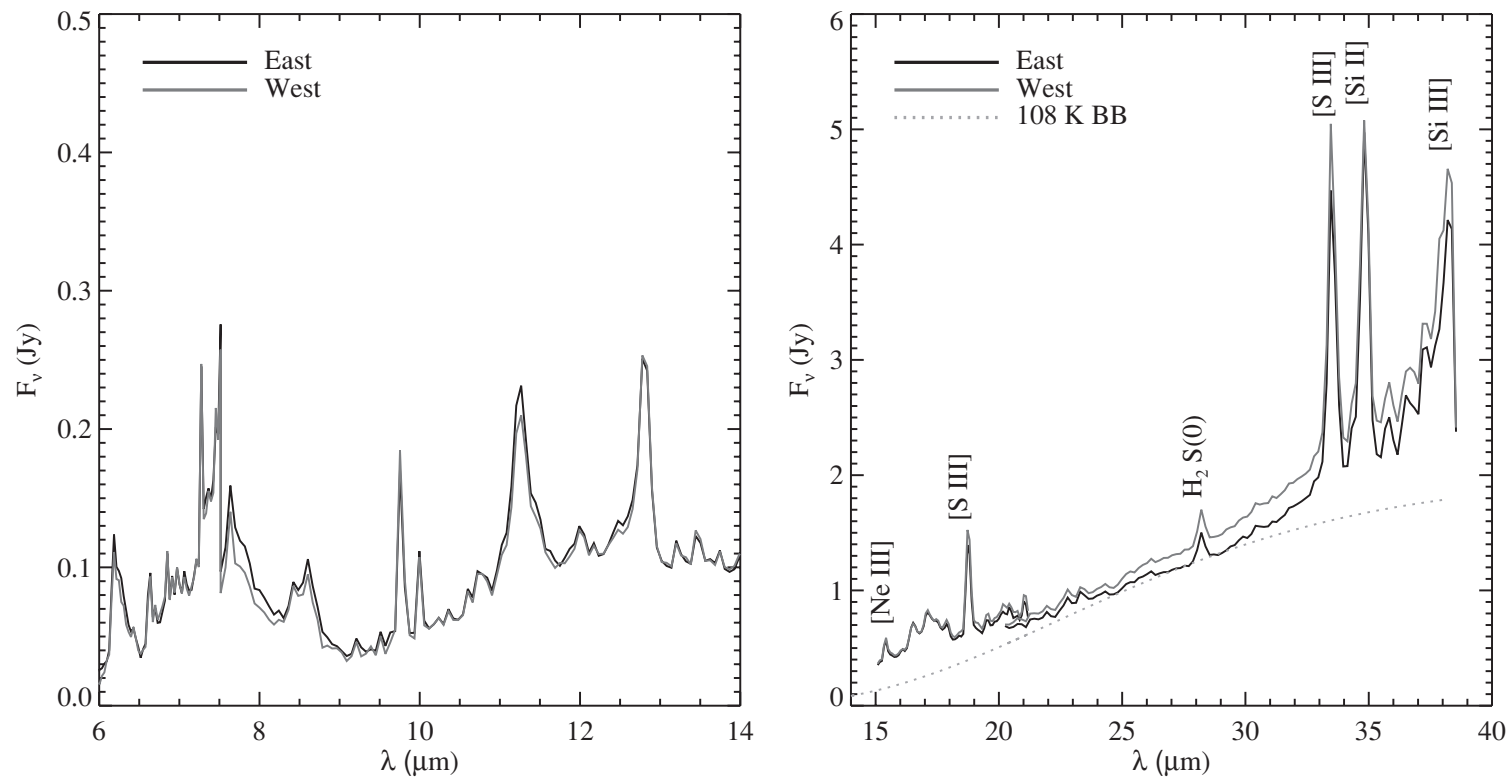

Figure 5. Left: SL IRS spectra from the two lobes, east in black and west in gray. Right: LL IRS spectra from the two lobes, east in black and west in gray. The labeled emission features likely belong to the fore/background Cygnus-X cloud, not BD+43 3710, as discussed in the text. A $108 \mathrm{~K}$ graybody is the "best" fit to the LL data for both lobes and is shown as the gray dotted line.

is little extended emission in the $5.8 \mu \mathrm{m}$ data that would have contained the $6.2 \mu \mathrm{m}$ feature if it were present at any significant strength.

Figure 8 shows a slice through the ring at position angle $30^{\circ}$, which corresponds to the narrowest point in the ring. The sharper, southwest edge of the ring is $\sim 1^{\prime}$ from the central star, or about $1.9 \mathrm{pc}$, assuming a distance of $6.5 \mathrm{kpc}$ (see modeling discussion below). The ring is more extended to the northeast, $\sim 1$.'2, or about $2.3 \mathrm{pc}$, and the edge is less well defined. Two possible explanations come to mind. First, the surrounding medium could be less dense to the northeast, allowing the shell to expand more easily in that direction. However, the $5.8 \mu \mathrm{m}$ data indicate that if anything there is more material to the east-northeast than toward the southwest. A second possibility is that the star is moving toward the southwest and the material on the "front" side of the shell is being compressed. Indeed, the proper motion of the HBHA 4202-22 is $\mu_{\alpha}=-4.5 \mathrm{mas} \mathrm{yr}^{-1}$, $\mu_{\delta}=-2.7$ mas yr$^{-1}$ (Zacharias et al. 2004), which corresponds to a position angle of $31^{\circ}$, nicely consistent with the compression direction. The distance to this object combined with the proper motion suggest that it may be a runaway star.

We used a modified version of the radiative transfer code of Egan et al. (1988) to model the dust shells around HBHA 4202-22. We modeled the central star as an A0 supergiant with $T_{*}=10,000 \mathrm{~K}, L_{*}=10^{5} L_{\odot}$ at a distance of $6.5 \mathrm{kpc}$, i.e., well behind the Cygnus- $\mathrm{X}$ region. While these parameters are formal inputs to the model, they, too, were varied in order to get the best fit to the observed data. As noted above, the shell 


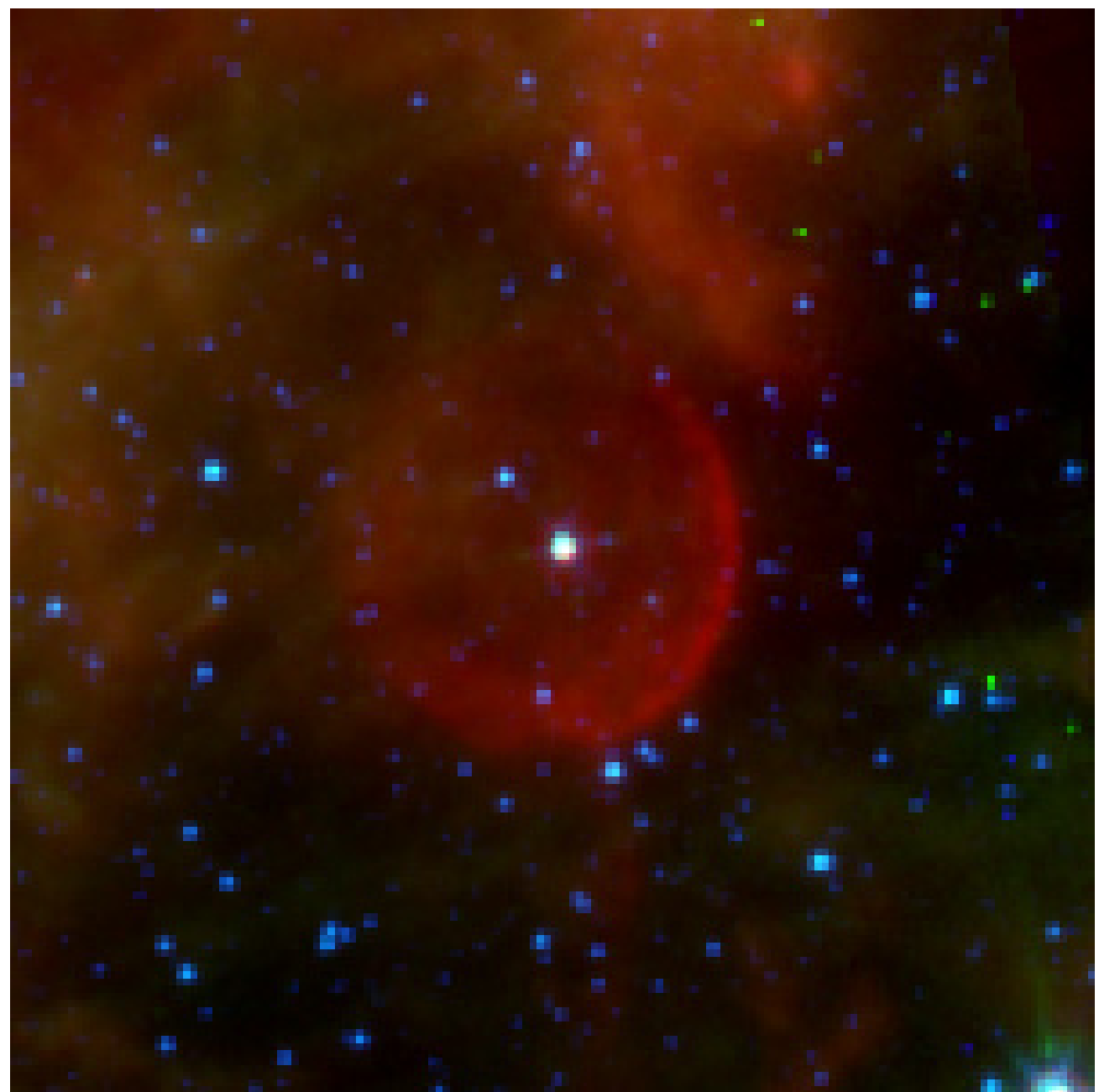

Figure 6. Three-color image of HBHA 4202-22. Red: $24 \mu \mathrm{m}$; green: $5.8 \mu \mathrm{m}$; blue: $3.6 \mu \mathrm{m}$. The image is $6^{\prime} .02 \times 6^{\prime} .02$ or $\sim 11.4 \mathrm{pc}$ at a distance of $6.5 \mathrm{kpc}$ (see the text).

around HBHA 4202-22 is compressed toward the southwest compared to the northeast. Therefore, we fit two models to the two portions. There is also a hint of a ring at $\sim 35^{\prime \prime}$ in the $5.8 \mu \mathrm{m}$ data, so an inner shell is also included in the model. Table 4 gives the derived parameters using amorphous carbon, with a particle size of $0.135 \mu \mathrm{m}$, an opacity of $\tau_{8.035 \mu \mathrm{m}}=7.5 \times 10^{-6}$ $\left(\tau_{0.55 \mu \mathrm{m}}=1.77 \times 10^{-2}\right)$, and grain constants from Mathis \& Whiffen (1988). Figures 9 and 10 show the results of the model compared to the data for the primary shells (compressed and not) at $24 \mu \mathrm{m}$. Models using silicate-rich dust were also considered, which result in cooler dust grains. The match to the data was not as good, though, so only the carbon-rich results are presented in Table 4.

\section{3. $G 79.29+0.46$}

Figure 11 shows the three-color image of G79.29+0.46 from the 3.6, 8, and $24 \mu \mathrm{m}$ data. It was first found to have a $\sim 4^{\prime}$ circumstellar ring in the radio by Higgs et al. (1994), who also suggested that the central object is probably an LBV. Subsequently, the same ring was detected in the infrared at a number of wavelengths from 8 to $60 \mu \mathrm{m}$ with IRAS (Waters et al. 1996), the Infrared Space Observatory (ISO; Wendker et al. 1998), and MSX (Egan et al. 2002; Clark et al. 2003). This inner ring is detected with all four IRAC bands as well as the $24 \mu \mathrm{m}$ MIPS band. Figure 12 shows the individual images from
Table 4

Dust Shell Parameters: HBHA 4202-22

\begin{tabular}{lcc}
\hline \hline Primary Shell & Compressed & Extended \\
\hline Inside radius & $5.28 \times 10^{18} \mathrm{~cm}$ & $5.28 \times 10^{18} \mathrm{~cm}$ \\
Thickness & $0.65 \times 10^{18} \mathrm{~cm}$ & $3.4 \times 10^{18} \mathrm{~cm}$ \\
Dust density law & $r^{-4}$ & $r^{0}$ \\
Dust temperature & $156.0-151.2 \mathrm{~K}$ & $156.0-137.4 \mathrm{~K}$ \\
Dust opacity & $\tau_{8.035 \mu \mathrm{m}=1.4 \times 10^{-4}}$ & \\
& $\tau_{0.55} \mu \mathrm{m}=1.77 \times 10^{-2}$ \\
\hline Inner shell & & \\
\hline Inside radius & $5 \times 10^{5} R_{*}$ & \\
& $3.70 \times 10^{18} \mathrm{~cm}$ & \\
Thickness & $0.70 \times 10^{18} \mathrm{~cm}$ & $r^{-2}$ \\
Dust density law & $196.6-188.2 \mathrm{~K}$ & \\
Dust temperature & $\tau_{8.035} \mu \mathrm{m}=7.5 \times 10^{-6}$ \\
Dust opacity & & \\
\hline
\end{tabular}

all five Spitzer bands. The apparent break in the south of the ring, particularly in the IRAC bands, is actually due to an infrared dark cloud (IRDC) that extends south-southwest in the figures. This suggests that as with HBHA 4202-22, G79.29+0.46 may be more distant than the $1.7 \mathrm{kpc}$ assumed for the Cygnus-X complex. Interestingly, though, the $\mathrm{CO}$ observations of Rizzo et al. (2008) also show a break in the CO emission in roughly 


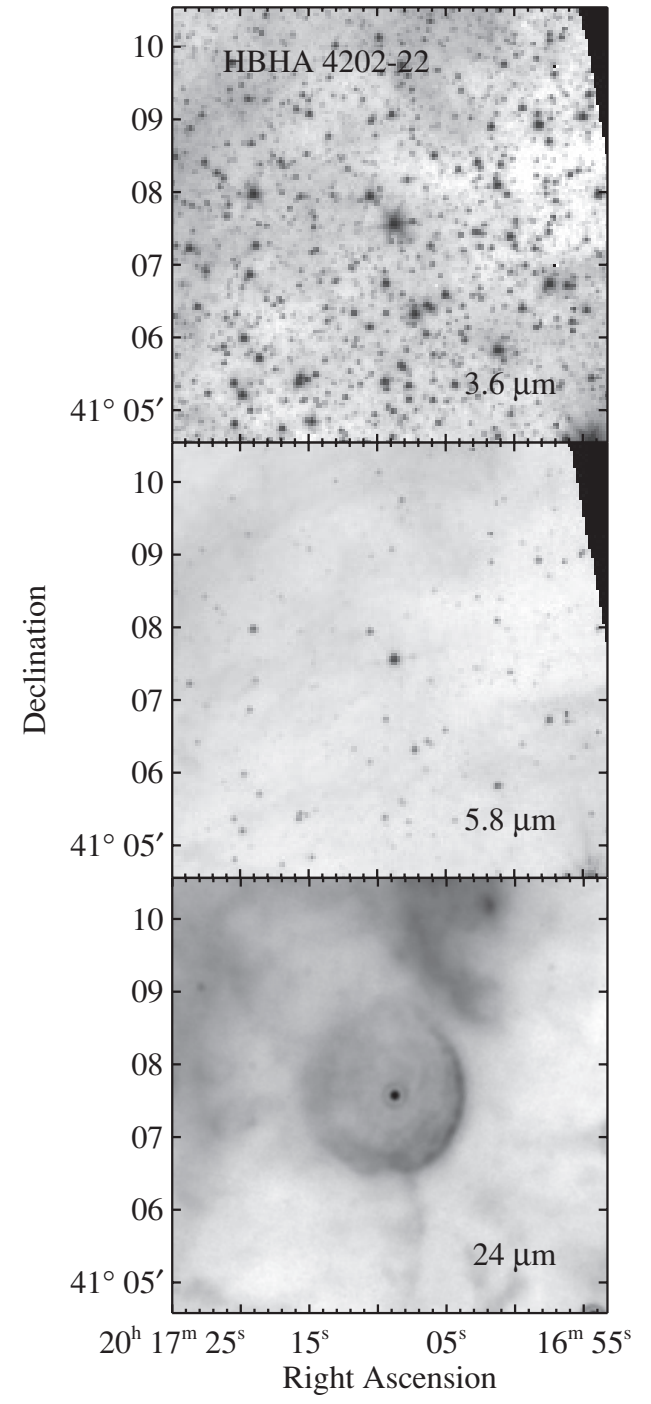

Figure 7. Individual images for HBHA 4202-22. As with BD+43 3710, there is no indication of extended emission associated with the star in the IRAC bands (top and middle panels), in contrast to the circumstellar shell detected at $24 \mu \mathrm{m}$ (bottom).

the same position as the IRDC. Since IRDCs are not known to absorb molecular line emission in the submillimeter, this suggests that the two structures might actually be interacting in some fashion. Additionally, the infrared ring appears to be somewhat flattened in the southeast, also consistent with possible interaction.

Also visible in the $24 \mu \mathrm{m}$ data in Figures 11 and 12 is a newly discovered outer ring $\sim 7^{\prime}$ across. This large ring probably represents an earlier episode of mass loss from G79.29+0.46 compared to the previously known inner ring. Additionally, the $24 \mu \mathrm{m}$ emission from the inner ring is much more extended than that from the shorter wavelengths. This kind of structure, where the IRAC emission ring is interior to a larger round emission structure at $24 \mu \mathrm{m}$, is occasionally seen in the MIPSGAL catalog of $24 \mu \mathrm{m}$ rings and disks (Mizuno et al. 2010). Usually, the $8 \mu \mathrm{m}$ emission appears to be co-spatial with the $24 \mu \mathrm{m}$ emission, if present, but most often it is absent entirely (as may be the case with HBHA 4202-22).

As with HBHA 4202-22, we modeled the dust shells for G79.29+0.46. The stellar parameters are $T_{*}=18,000 \mathrm{~K}$, $L_{*}=4 \times 10^{5} L_{\odot}, R_{*}=4.567 \times 10^{12} \mathrm{~cm}$, with a distance

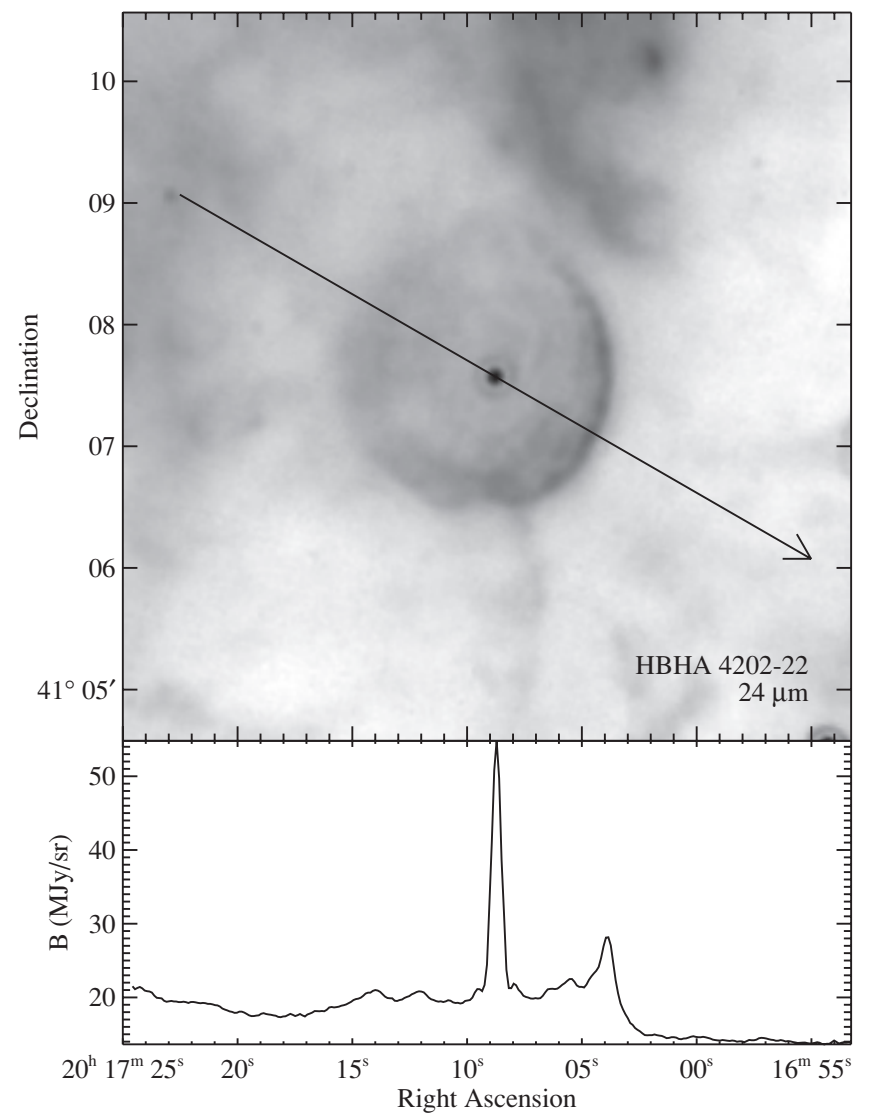

Figure 8. Top: $24 \mu \mathrm{m}$ ring around HBHA 4202-22. Bottom: slice through the $30^{\circ}$ position angle indicated by the line in the top figure. This angle, which corresponds to the narrowest part of the ring, is just $1^{\circ}$ off of the $31^{\circ}$ propermotion direction.

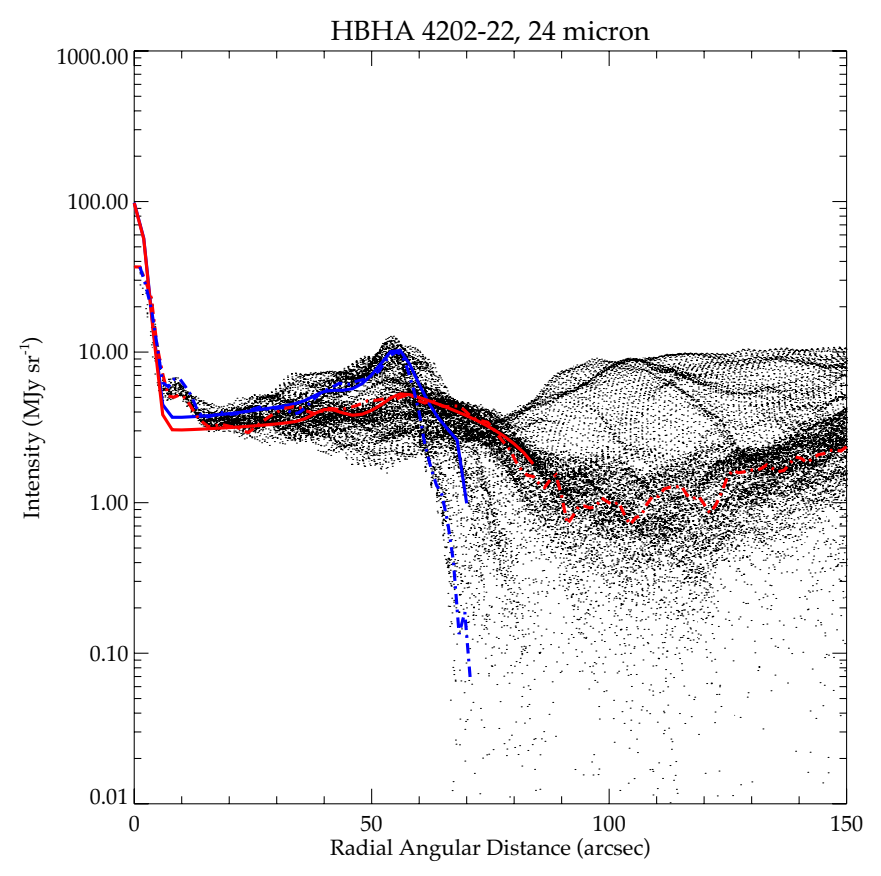

Figure 9. Radial profiles for HBHA 4202-22. The dots show the $24 \mu \mathrm{m}$ data; the dash-dotted lines show a horizontal slice through the data, red for the extended side, blue for the compressed side. The solid colored lines show the model results.

(A color version of this figure is available in the online journal.) 


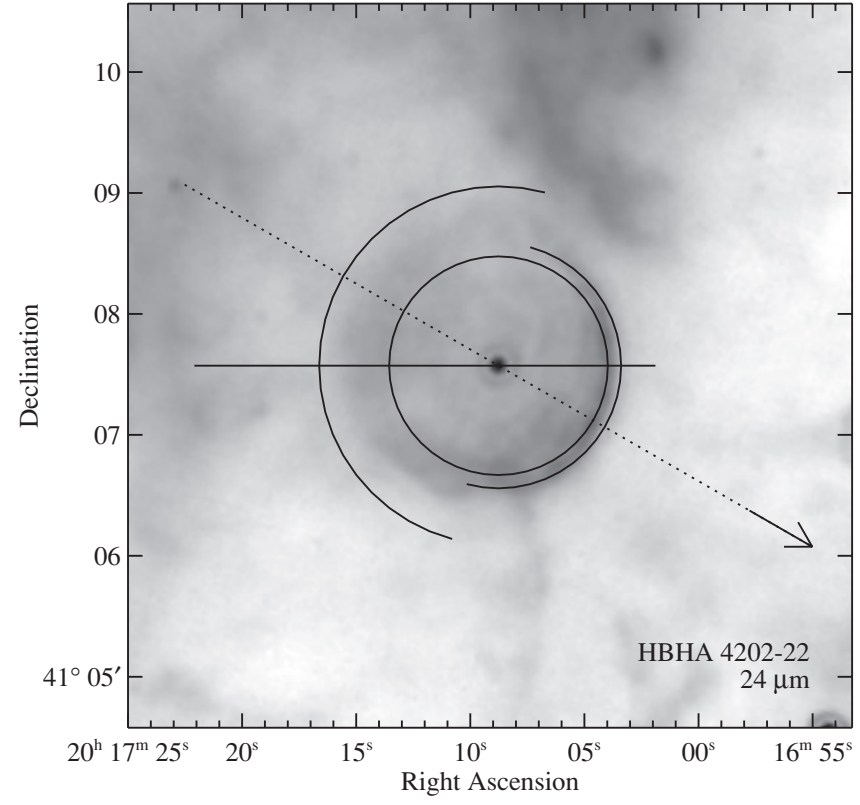

Figure 10. Approximate range of the extended and compressed shells. Arcs showing the extent of the dust models superposed on the $24 \mu \mathrm{m}$ image. Their angular extent was estimated by eye, not fit by the model. The solid horizontal line indicates the radii shown with the red and blue lines in Figure 9. The dotted line with the arrow indicates the direction of the proper motion of HBHA 4202-22. of $d=9.25 \times 10^{21} \mathrm{~cm}$, i.e., slightly behind Cygnus- $\mathrm{X}$ at $\sim 3 \mathrm{kpc}$. Table 5 lists the derived parameters for the inner and outer shells. A mix of amorphous carbon grains and PAHs (Li \& Draine 2001) was used in the model, as the dust alone could not produce the flux detected in the IRAC bands and the strong PAH emission seen in the IRS observations of G. Umana et al. (2010, in preparation). ${ }^{14}$ The amorphous carbon grains dominate the mass and opacity, although the (much) smaller PAHs are more numerous. While the inner shell is modeled as a single shell with a density law of $r^{-3.5}$, the $24 \mu \mathrm{m}$ flux profile (Figure 13) suggests that a more complex model with a different density law for the interior of the shell might give better results.

\section{SUMMARY}

We have detected extended $24 \mu \mathrm{m}$ emission around three evolved stars in the Cygnus-X Legacy survey region. A bipolar nebula was found around the carbon star BD+43 3710. IRS spectra show that the emission is due to small dust grains, rather than [O IV] line emission or the broad $\mathrm{MgS}$ feature. An asymmetric ring of $24 \mu \mathrm{m}$ emission was observed around the Wolf-Rayet candidate HBHA 4202-22. The "compressed"

14 Morris et al. (2008) present an infrared spectrum of G79.29+0.46 that included IRS data as well as ISOSWS and near-infrared spectra. Presumably the IRS spectrum is from the central star as it does not show the strong PAHs that are detected by G. Umana et al. (2010, in preparation) toward the shell.

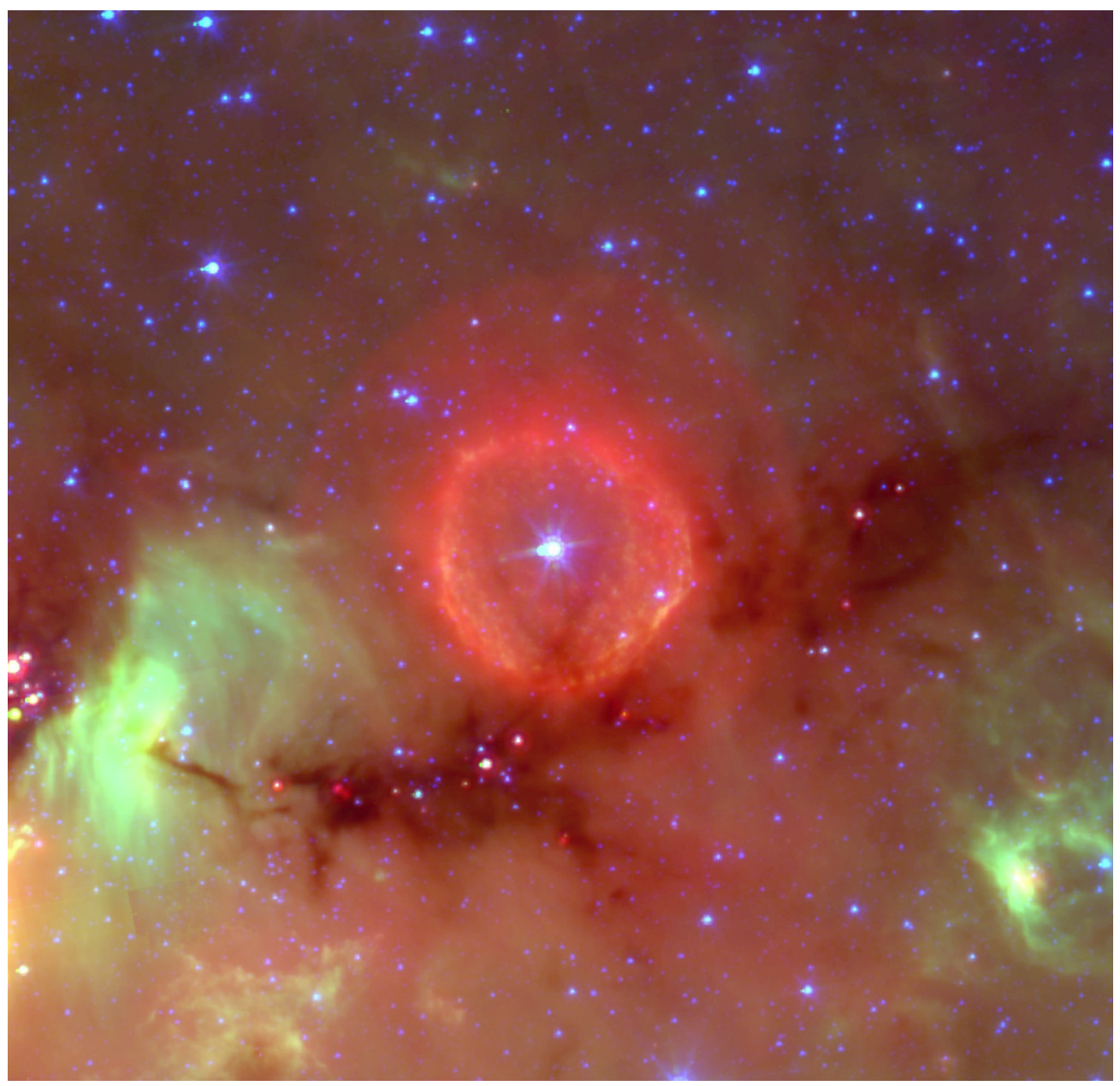

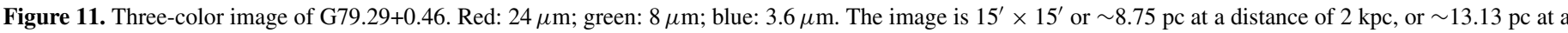
distance of $3 \mathrm{kpc}$ (see the text). 


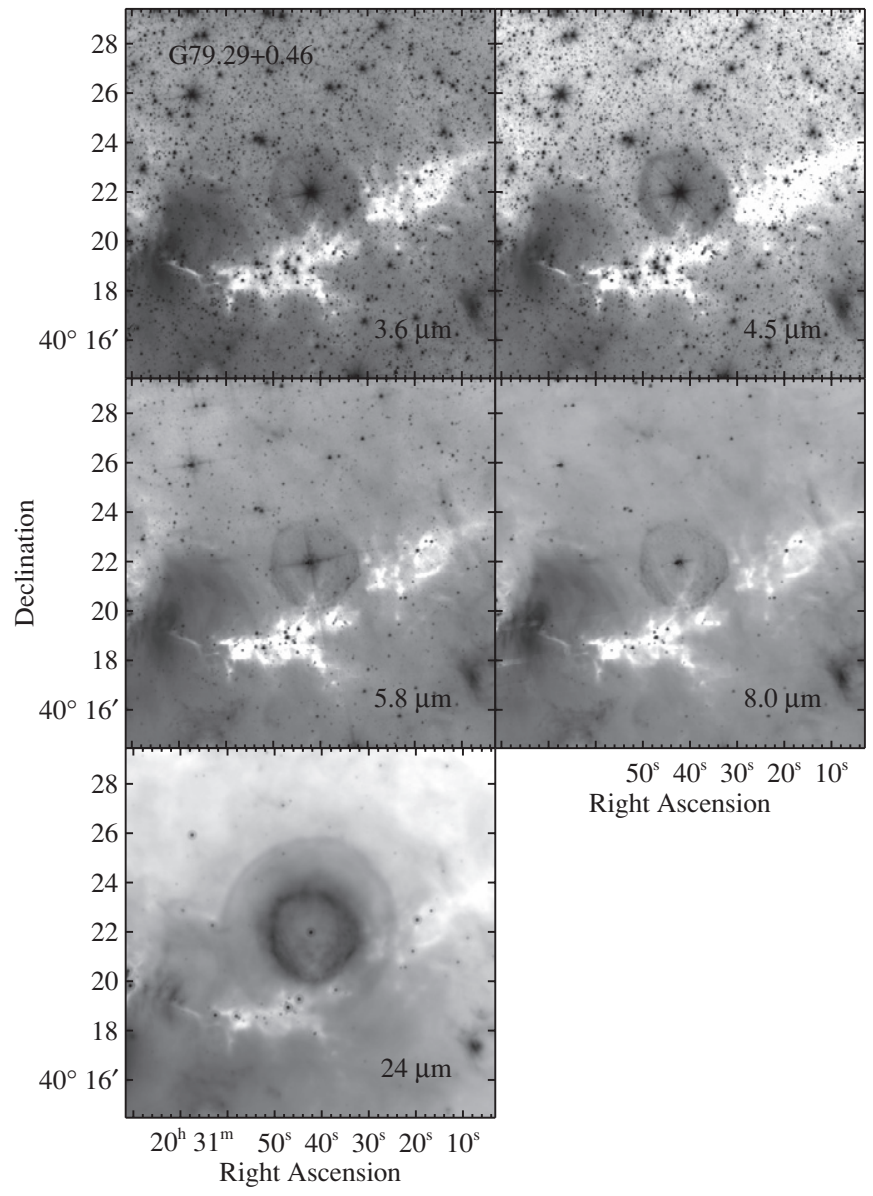

Figure 12. Individual images of G79.29+0.46 in the IRAC bands (top two rows) and at $24 \mu \mathrm{m}$ (bottom). Note that the gap in the south of the inner ring is caused by foreground absorption associated with the IRDC stretching across the bottom of the images. Grayscales are logarithmic.

portion of the ring is in the direction of the projected velocity vector derived from the observed proper motion of the star. Radiative transfer models imply that the central star is an A-type supergiant at a distance of $\sim 6.5 \mathrm{kpc}$ and the ring is composed of amorphous carbon grains. A large, $\sim 7^{\prime}$ diameter ring was detected at $24 \mu \mathrm{m}$ around the LBV candidate G79.29+0.46, a new addition to the well-known inner ring. The inner ring was detected at all four IRAC bands as well as at $24 \mu \mathrm{m}$. The shells were modeled with a mixture of amorphous carbon grains and hot PAHs. An IRDC blocks part of the ring emission at the shorter wavelengths, which along with the model results indicates that the LBV candidate is at least on the far side of the Cygnus- $X$ region, if not further.

While somewhat disparate in their properties, these three objects demonstrate the potential of the Spitzer data archives. The thrust of the Cygnus-X Legacy project was to explore the richest high-mass star formation region within $2 \mathrm{kpc}$. Similarly, the larger Spitzer Legacy programs that mapped the inner Galactic plane with IRAC (GLIMPSE, the Galactic Legacy Infrared Mid-Plane Survey Extraordinaire; Benjamin et al. 2003) and MIPS (MIPSGAL; Carey et al. 2009) also emphasized star formation studies in their proposals and overview papers. In each case, though, a wealth of information on circumstellar structures around evolved stars also has been obtained. For example, hundreds of small $24 \mu \mathrm{m}$ emission rings have been found in the MIPSGAL survey data (Mizuno et al. 2010), many of which are similar to the structures around HBHA 4202-22

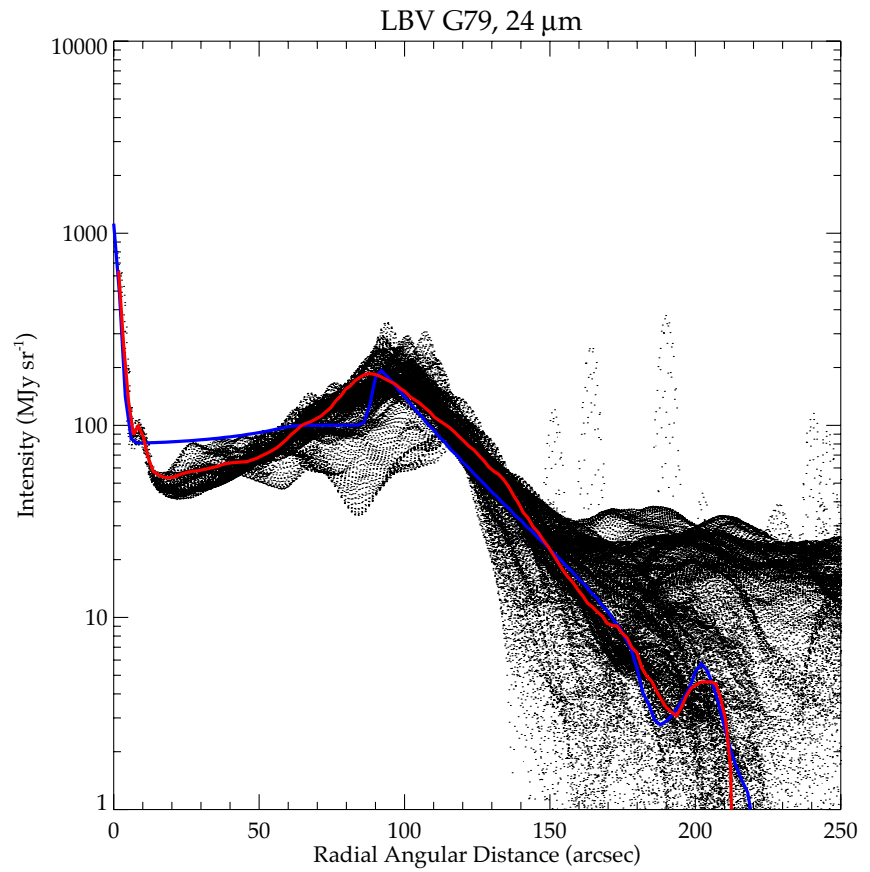

Figure 13. Radial profiles of the rings around G79.29+0.46. The red line is a slice through the data westward of the central star. The blue line shows the model fit.

(A color version of this figure is available in the online journal.)

Table 5

Dust Shell Parameters: G79.29+0.46

\begin{tabular}{lcc}
\hline \hline \multicolumn{1}{c}{ Parameters } & Inner Shell & Outer Shell \\
\hline Inside radius & $3.973 \times 10^{18} \mathrm{~cm}$ & $8.99 \times 10^{18} \mathrm{~cm}$ \\
& $8.7 \times 10^{5} R_{*}$ & $1.1 R_{\text {inner }}$ \\
Thickness & $4.2 \times 10^{18} \mathrm{~cm}$ & $0.5 \times 10^{18} \mathrm{~cm}$ \\
Dust density law & $r^{-3.5}$ & $r^{-2}$ \\
Dust temperature & & $87.75-86.57 \mathrm{~K}$ \\
Am. carbon & $107.6-89.9 \mathrm{~K}$ & $96.1-94.4 \mathrm{~K}$ \\
PAHs & $1500-354 \mathrm{~K}$ & $0.40 \mu \mathrm{m}$ \\
Dust components & & $5.0 \times 10^{-4} \mu \mathrm{m}$ \\
$a_{\text {am.car. }}$ & $0.45 \mu \mathrm{m}$ & $3 \%$ \\
$a_{\text {PAHs }}$ & $5.0 \times 10^{-4} \mu \mathrm{m}$ & $97 \%$ \\
$N_{\text {am.car. }}$ & $2 \%$ & $9.81 \times 10^{-6}$ \\
$N_{\text {PAHs }}$ & $98 \%$ & $2.00 \times 10^{-4}$ \\
Dust opacity & & $9.39 \times 10^{-4}$ \\
$\tau_{24} \mu \mathrm{m}$ & $1.324 \times 10^{-4}$ & \\
$\tau_{8.0} \mu \mathrm{m}$ & $2.70 \times 10^{-3}$ & \\
$\tau_{0.55} \mu \mathrm{m}$ & $1.27 \times 10^{-2}$ & \\
\hline
\end{tabular}

Notes.

a PAH temperature is non-equilibrium for the inner shell and declines as $r^{-2}$. The outer shell is an equilibrium temperature.

b $a=$ grain size; $N=$ number density within the shell.

and G79.29+0.46 (the main difference being that most of the MIPSGAL rings have no detected $24 \mu \mathrm{m}$ central source). As with our two ring sources, those objects that have previous identifications are often identified as emission line stars. It is also interesting to note that we detect extended emission in the IRAC bands only toward the inner ring of G79.29+0.46, and the MIPSGAL rings have IRAC counterparts less than $15 \%$ of the time. Data from follow-up IRS observations are currently being analyzed to determine what mechanism is responsible for the $24 \mu \mathrm{m}$ emission in a selection of the MIPSGAL rings ( $\mathrm{N}$. Flagey et al. 2010, in preparation)_amorphous carbon, as in 
BD+43 3710; [O IV] as in the Morris et al. and Billot et al. SNRs; the as-yet-unidentified $21 \mu \mathrm{m}$ feature seen in post-AGB stars; or some other mechanism. Results from these, as well as other studies underway, will enable us to better place our three objects in the broader context of evolved stars with circumstellar envelopes in the Galaxy.

Note added in proof. We note the following papers were published during the review process which may be of interest to the reader, as they discuss additional aspects of G79.29+0.46 and HBHA 4202-22, respectively: Jiménez-Esteban et al. 2010, ApJ, 713, 429 and Gvaramadze et al. 2010, MNRAS, in press; and Gvaramadze et al. 2009, MNRAS, 400, 524.

This work is based in part on observations made with the Spitzer Space Telescope, which is operated by JPL/Caltech under NASA contract 1407. Support for this work was provided in part by NASA. This research has made use of NASA's Astrophysics Data System Bibliographic Services, data products from 2MASS which is a joint project of the University of Massachusetts and IPAC/Caltech funded by NASA and the NSF, and the Simbad database operated at CDS, Strasbourg, France. We thank the anonymous referee for useful suggestions that improved the paper.

Facilities: Spitzer(IRAC, IRS, MIPS)

\section{REFERENCES}

Aoki, W., Tsuji, T., \& Ohnaka, K. 1998, A\&A, 340, 222

Argelander, F. W. A. 1903, in Bonner Durchmusterung, ed. A. Marcus \& E. Weber (Bonn: Verlag)

Benjamin, R. A., et al. 2003, PASP, 115, 953

Bernard-Salas, J., Peeters, E., Sloan, G. C., Gutenkunst, S., Matsuura, M., Tielens, A. G. G. M., Zijlstra, A. A., \& Houck, J. R. 2009, ApJ, 699, 1541

Billot, N., Flagey, N., Noriega-Crespo, A., Shenoy, S., Carey, S., Mizuno, D., Kraemer, K., \& Latter, W. 2009, BAAS, 214, 316.05

Carey, S. J., et al. 2009, PASP, 121, 76

Clark, J. S., Egan, M. P., Crowther, P. A., Mizuno, D. R., Larionov, V. M., \& Arkharov, A. 2003, A\&A, 412, 185

Cutri, R. M., et al. 2003, The IRSA 2MASS All-Sky Point Source Catalog, NASA/IPAC Infrared Science Archive (http://irsa.ipac.caltech.edu/ applications/Gator/)

Dolidze, M. V. 1971, Astron. Tsirk., 629, 6

Egan, M. P., Clark, J. S., Mizuno, D. R., Carey, S. J., Steele, I. A., \& Price, S. D. 2002, ApJ, 572, 288

Egan, M. P., Leung, C. M., \& Spagna, G. F., Jr. 1988, Comput. Phys. Commun., 48, 271
Egan, M. P., et al. 2003, Air Force Research Laboratory Technical Report AFRL-VS-TR-2003-1589, ADA418993

Engelbracht, C. W., et al. 2007, PASP, 119, 994

Fazio, G. G., et al. 2004, ApJS, 154, 10

Forrest, W. J., Houck, J. R., \& McCarthy, J. F. 1981, ApJ, 248, 195

Goebel, J. H., Bregman, J. D., Cooper, D. M., Goorvitch, D., Langhoff, S. R., \& Witteborn, F. C. 1983, ApJ, 270, 190

Goebel, J. H., Bregman, J. D., Strecker, D. W., Witteborn, F. C., \& Erickson, E. F. 1978, ApJ, 222, L129

Goebel, J. H., \& Moseley, S. H. 1985, ApJ, 290, L35

Gutermuth, R. A., et al. 2008, ApJ, 674, 336

Gvaramadze, V. V., et al. 2009, MNRAS, 400, 524

Gvaramadze, V. V., et al. 2010, MNRAS, in press

Herbig, G. H. 2009, AJ, 138, 1502

Higgs, L. A., Wendker, H. J., \& Landecker, T. L. 1994, A\&A, 291, 295

Høg, E., et al. 2000, A\&A, 355, L27

Hony, S., Waters, L. B. F. M., \& Tielens, A. G. G. M. 2002, A\&A, 390, 533

Hora, J. L., et al. 2008a, PASP, 120, 1233

Hora, J. L., et al. 2008b, New Light on Young Stars: Spitzer's View of Circumstellar Disks (http://www.ipac.caltech.edu/spitzer2008/proceedings.html)

Houck, J. R., et al. 2004, ApJS, 154, 18

Jiménez-Esteban, F. M., Rizzo, J. R., \& Palau, A. 2010, ApJ, 713, 429

Kohoutek, L., \& Wehmeyer, R. 1999, A\&AS, 134, 255

Kraemer, K. E., Sloan, G. C., Price, S. D., \& Walker, H. J. 2002, ApJS, 140, 389

Kraemer, K. E., et al. 2009, BAAS, 41, 467

Li, A., \& Draine, B. T. 2001, ApJ, 554, 778

Mathis, J. S., \& Whiffen, G. 1988, ApJ, 341, 808

Mizuno, D. R., et al. 2008, PASP, 120, 1028

Mizuno, D. R., et al. 2010, AJ, 139, 1542

Morris, P. W., Crowther, P. A., \& Houck, J. R. 2004, ApJS, 154, 413

Morris, P., \& the Spitzer WRRINGS Team, 2008, IAU Symp. 250, Massive Stars as Cosmic Engines, ed. F. Bresolin, P. A. Crowther, \& J. Puls (Cambridge: Cambridge Univ. Press), 361

Morris, P. W., et al. 2006, ApJ, 640, L179

Nassau, J. J., \& Blanco, V. M. 1954, ApJ, 120, 129

Rieke, G. H., et al. 2004, ApJS, 154, 25

Rizzo, J. R., Jimenez-Estaban, F. M., \& Ortiz, E. 2008, ApJ, 681, 355

Schneider, N., Bontemps, S., Simon, R., Jokob, H., Motte, F., Miller, M., \& Kramer, C. 2006, A\&A, 458, 855

Schneider, N., Simon, R., Bontemps, S., Comerón, F., \& Motte, F. 2007, A\&A, 474,873

Skrutskie, M. F., et al. 2006, AJ, 131, 1163

Sloan, G. C., Kraemer, K. E., Price, S. D., \& Shipman, R. F. 2003, ApJS, 147, 379

Voors, R. H. M., Geballe, T. R., Waters, L. B. F. M., Najarro, F., \& Lamers, H. L. G. L. M. 2000, A\&A, 362, 236

Waters, L. B. F. M., Izumiura, H., Zaal, P. A., Geballe, T. R., Kester, D. J. M., \& Bontekoe, T. R. 1996, A\&A, 313, 866

Wendker, H. J., Molthagen, K., Heske, A., Higgs, L. A., \& Landecker, T. L. 1998, Ap\&SS, 255, 187

Werner, M., et al. 2004, ApJS, 154, 1

Zacharias, N., Urban, S. E., Zacharias, M. I., Wycoff, G. L., Hall, D. M., Germain, M. E., Holdenried, E. R., \& Winter, L. 2004, AJ, 127, 3043

Zijlstra, A. A., et al. 2006, MNRAS, 370, 1961 